\title{
The Value of 2D Strain Imaging during Stress Testing
}

\author{
Marie Moonen, M.D., Patrizio Lancellotti, M.D., Ph.D., Dimitrios Zacharakis, M.D., and Luc Pierard, M.D.,
} Ph.D., F.E.S.C., F.A.C.C.

CHU Sart Tilman, Department of Cardiology, Liege, Belgium

\begin{abstract}
Stress echocardiography is increasingly used but its major limitation is the subjective interpretation of wall motion changes requiring experience. Speckle tracking enables simultaneous evaluation of radial, longitudinal, and circumferential myocardial deformation. Recently, two-dimensional (2D) strain has been found to be as reliable as sonomicrometry for the assessment of left ventricular (LV) regional function. In the presence of inducible ischemia, longitudinal and circumferential abnormalities preceed the decrease in radial deformation. Optimal cutoffs have been obtained from 2D strain rate (SR) at peak dobutamine stress to predict coronary artery disease. However, 2D strain rate does not yet provide incremental accuracy to visual interpretation by experts. Speckle tracking strain could be useful to better identify contractile reverse and biphasic response of viable myocardium but there are not yet clinical studies published in this setting. Preliminary results suggest that 2D strain obtained during exercise could be useful in asymptomatic patients with severe aortic stenosis or organic mitral regurgitation (MR). In conclusion, the reliability and clinical importance of 2D strain during stress will be specified by further investigations.
\end{abstract}

Keywords: dobutamine stress echocardiography, exercise echocardiography, ischemic cardiomyopathy, ischemic heart disease, strain, strain rate imaging

Stress echocardiography is nearly 30 years old. ${ }^{1}$ The following major steps have improved its accuracy and acceptance: synchronized display of the different stages, use of pharmacologic stressors, such as dobutamine and dipyridamole, ${ }^{2,3}$ improved endocardial detection by tissue harmonic imaging ${ }^{4}$ and left ventricular (LV) enhancement by contrast agents ${ }^{5}$ and more recently the introduction of quantitative assessment of regional function. ${ }^{6}$ Most stress echo laboratories still analyze recordings by the conventional visual "eyeball" assessment of changes in timing and amplitude of endocardial motion and myocardial thickening. A major drawback is the subjective interpretation requiring dedicated training and experience. ${ }^{7}$ Moderate reproducibility has been observed with dobutamine echocardiography, even by experts. ${ }^{8,9}$ The level of agreement has not been evaluated for exercise echocardiography.

The need of a more quantitative method for the interpretation of stress echocardiography has depended on the development of new imaging modalities. Color kinesis permits quantification of endocardial motion, but not of myocardial thickening and has a too low temporal resolution. ${ }^{10}$ Tissue Doppler imaging and derived strain and strain rate (SR) measurements have offered alternatives for better quantification of regional contraction at rest or during stress. ${ }^{11,12}$ Tissue Doppler is feasible during stress testing but requires a high frame rate of at least 140 frames s${ }^{-1}$. Another major limitation of tissue Doppler is that peak velocity and strain variables depend on the angle between the incident ultrasound beam and the myocardial wall. ${ }^{13}$ These constraints limit imaging in the apical projections and require different image processing techniques that are challenging and time consuming, thus reducing a wide adoption of this method. Finally, the complex three-dimensional (3D) deformation of the heart during the cardiac cycle may not be adequately captured only in the longitudinal direction.

More recently, 2D strain, which is not Doppler based but based on speckle tracking, offers promising alternative. ${ }^{14}$ This new technique is not influenced by the angle of the incidence of the ultrasound beam ${ }^{15,16}$ and can be potentially used in any myocardial section without paying too much attention to the orientation of the heart. The tracking system is based on gray-scale B-mode images. The deformation is obtained by automatic tracking, during the cardiac cycle of the distance between two pixels of a myocardial segment. In fact, a large number of small regions are tracked and a software averages their motions before extracting regional motion curves. The tracking quality can be verified for each segment, with subsequent manual adjustment of the region 
of interest, if necessary. This new method enables simultaneous evaluation of the 3 components of myocardial deformation: radial, longitudinal and circumferential. Practically, longitudinal strain and SR are obtained from the apical views, radial and circumferential strain and SR from the parasternal short-axis views Indeed, the distribution of myocardial fibers within the wall has clinical importance for the analysis of inducible ischemia. ${ }^{17}$

\section{D STRAIN FOR THE DETECTION OF ISCHEMIA}

Detection of ischemia when a coronary artery disease is suspected is essential. Exercise and dobutamine stress echocardiography (DSE) are well established for identifying inducible ischemia. Ischemia is defined by a regional reduction of myocardial thickening or abnormal wall motion. Myocardial ischemia can also provoke a delayed onset and termination of systolic thickening. Unfortunately, the human eye has insufficient temporal resolution to identify these abnormalities in real time.

Recently, Reant et al. ${ }^{18}$ investigated 2D strain applicability for the detection of ischemia during DSE. In a large animal open-chest model, myocardial deformation was studied before and during dobutamine infusion, under controlled ischemic conditions produced by various degrees of left anterior descending (LAD) coronary artery constriction. Four progressive stenoses were obtained inducing reduction of hyperemic flow by $40 \%$ and $70 \%$ and resting flow by $25 \%$ and $50 \%$, respectively. Two-dimensional strain echocardiography was compared to sonomicrometry: three pairs of segment-length ultrasonic crystals were inserted in the LV anterior wall to permit strain analysis of the three different components of myocardial motion. To investigate radial strain, one crystal was placed in the inner layer and another in the outer layer. To analyse longitudinal strain, crystals were placed parallel to the long axis of the left ventricle. For circumferential assessment, crystals were placed perpendicular to the long axis. Strain in these three directions was obtained by calculation of the instantaneous distance between crystals normalized to the end-diastolic length. The echocardiographic planes were matched to the crystals positions by direct visualization. Circumferential strain, radial strain and wall thickening were obtained in the parasternal short-axis view. Apical views permitted the measurement of longitudinal strain in the ischemic (anterior wall) and control areas (infer-olateral wall). The main findings of the study indicated that 2D strain is as reliable as sonomicrometry for the assessment of LV regional function. The authors also evaluated the three different strain components of myocardial deformation during DSE and demonstrated that in the presence of ischemia, longitudinal and circumferential abnormalities precede the decrease in radial deformation. They explained this observation by the fact that subendocardial myocardial fibers are mainly oriented longitudinally. As the subendocardial layer is more sensitive to ischemia, the longitudinal function could be altered earlier than the radial function. Indeed, longitudinal strain decreased at lower ischemic level and at lower doses of dobutamine than radial strain.

Recent analyses of the feasibility of the speckle tracking during DSE tested the clinical accuracy of this new technique. Ingul et al. ${ }^{19}$ investigated myocardial deformation during DSE in 197 patients by automated deformation analysis. All patients had normal wall motion score at rest. They described an automated method for analysis of myocardial deformation that is feasible and time-saving. The SR was measured by tissue Doppler, from the velocity gradient along a fixed distance along the ultrasound beam, and strain was measured by the temporal integration of SR. In the segment length method, strain was measured directly by changes in segment length along the direction of the wall, not along the ultrasound beam and thus was angle independent, and the SR was calculated as the temporal derivative of strain, with correction to Eulerian SR. The patients were separated into 3 groups: patients at low probability of coronary artery disease (Framingham risk score $<1 \% /$ year) who did not undergo coronary angiography, patients with normal angiography and patients with $>50 \%$ narrowing of at least one major vessel. Different parameters were obtained. Peak systolic SR was determined as the maximal negative SR value during ejection time, end-systolic strain as the magnitude of strain at aortic valve closure, and peak postsystolic strain as the greatest value after end systole. All segments supplied by a stenosed coronary artery were labelled "at risk," and the presence of a minimum peak systolic SR value less than the normal range defined the territory as abnormal. They determined the optimal cutoffs for peak SR range: $1.3 \mathrm{~s}^{-1}$ for the velocity gradient method and $1.2 \mathrm{~s}^{-1}$ for the segment length method. This study showed that the optimal parameters of stress-induced ischemia are peak systolic SR and end-systolic strain. The authors concluded that this mixed technique was feasible and accurate and could enhance sensitivity in the hands of expert investigators, both methods having comparable sensitivities and specificities.

Another interesting study by Hanekom et al. ${ }^{20}$ compared in 150 consecutive patients $2 \mathrm{D}$ strain by the speckle tracking method and tissue Doppler strain measurements during DSE, in correlation with coronary angiography. Two-dimensional strain and tissue velocity imaging-strain were obtained in three apical views (four-chamber, 
long-axis, and two-chamber views) at rest and peak stress. To avoid the requirement of measuring waveforms in all 16 segments and to make the technique feasible in daily practice, they studied single sentinel segments at the distal end of the perfusion territory of each coronary artery. For both methods, in the territory of the LAD artery, the optimal segment was the apical septum. In the posterior wall and in the right coronary artery territory, different segments were identified by 2D strain and tissue velocity imaging; they selected the segment that gave the best overall performance: basal posterior wall for the left circumflex and mid inferior segment for the right coronary artery territory. The diagnostic accuracy of 2D strain was lower than the accuracy of tissue Doppler in the right coronary territory, but was similar in the LAD coronary circulation. The sensitivity, specificity, and accuracy of 2D strain are higher in the LAD territory than in both the circumflex and the right coronary artery territories. The lower sensitivity for the detection of disease in the posterior-lateral circulation may be due to problems of image quality in these segments. The accuracy of 2D strain during tracking was dependent on the characteristics of the image, and because of its dependence on gray scale image quality, 2D strain seemed less reliable in the posterior circulation, with the worst performance in the anterolateral wall. In contrast, tissue velocity imaging based strain was less dependent on image quality and consequently constituted an alternative for the posterior coronary circulation. Using the sentinel segment, they compared the two strain measurement techniques and wall motion scoring with the presence of a significant coronary stenosis. Optimal cutoffs of both 2D SR and tissue Doppler imaging SR at peak stress were obtained to predict coronary artery disease (Table I). They found that neither 2D SR nor tissue Doppler imaging SR at peak stress provided incremental accuracy to visual interpretation by experts for diagnosis of significant coronary artery disease per patient. It should be remembered that for simplification reasons, strain was evaluated in only one representative segment for each coronary artery territory. This could have contributed to the relative lack of advantage of strain imaging. Figures 1 and 2 illustrate the incremental value of 2D strain (Fig. 1) and SR (Fig. 2) in a patient in whom the qualitative interpretation of DSE was difficult. Complete inversion of strain and SR curves is observed in both inferior and superior segments displayed in the apical two-chamber view. The coronary angiography showed significant stenoses of the right coronary artery and a diagonal branch of the LAD and diffuse atherosclerosis of the distal LAD.

Figure 1. Detection of dobutamine induced ischemia from strain curves at rest and during dobutamine test. Rest: similar values of strain in the anterior and inferior wall. Low dose: reduction of strain and peak postsystolic strain at midanterior wall. High dose: complete inversion of strain curves in both anterior and inferior walls. Coronary angiography revealed diffuse atherosclerosis of the distal LAD and severe stenoses of a diagonal branch and of the right coronary artery.

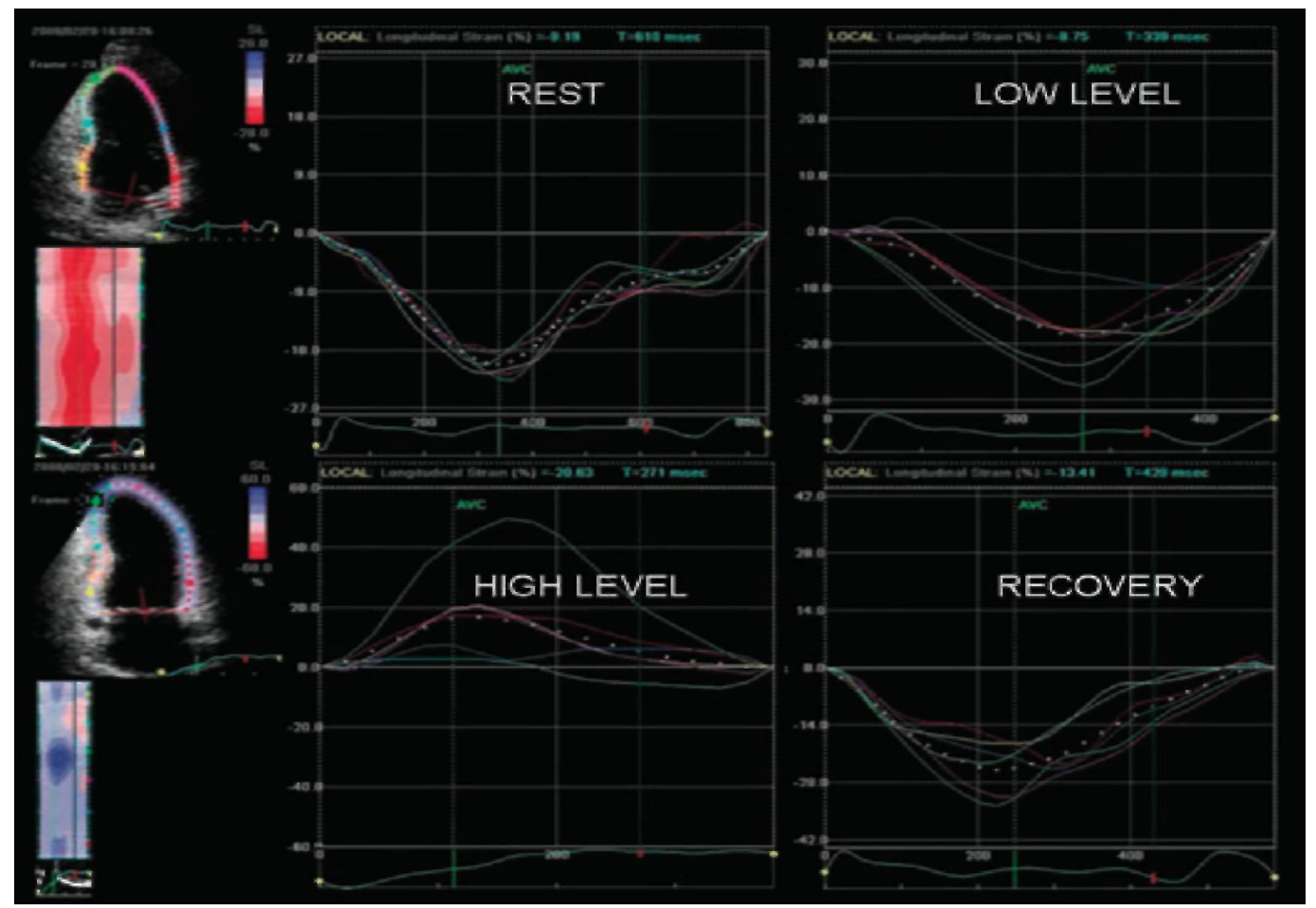


Figure 2. Strain rate curves at rest and during stress in the same patient as in Figure 1.

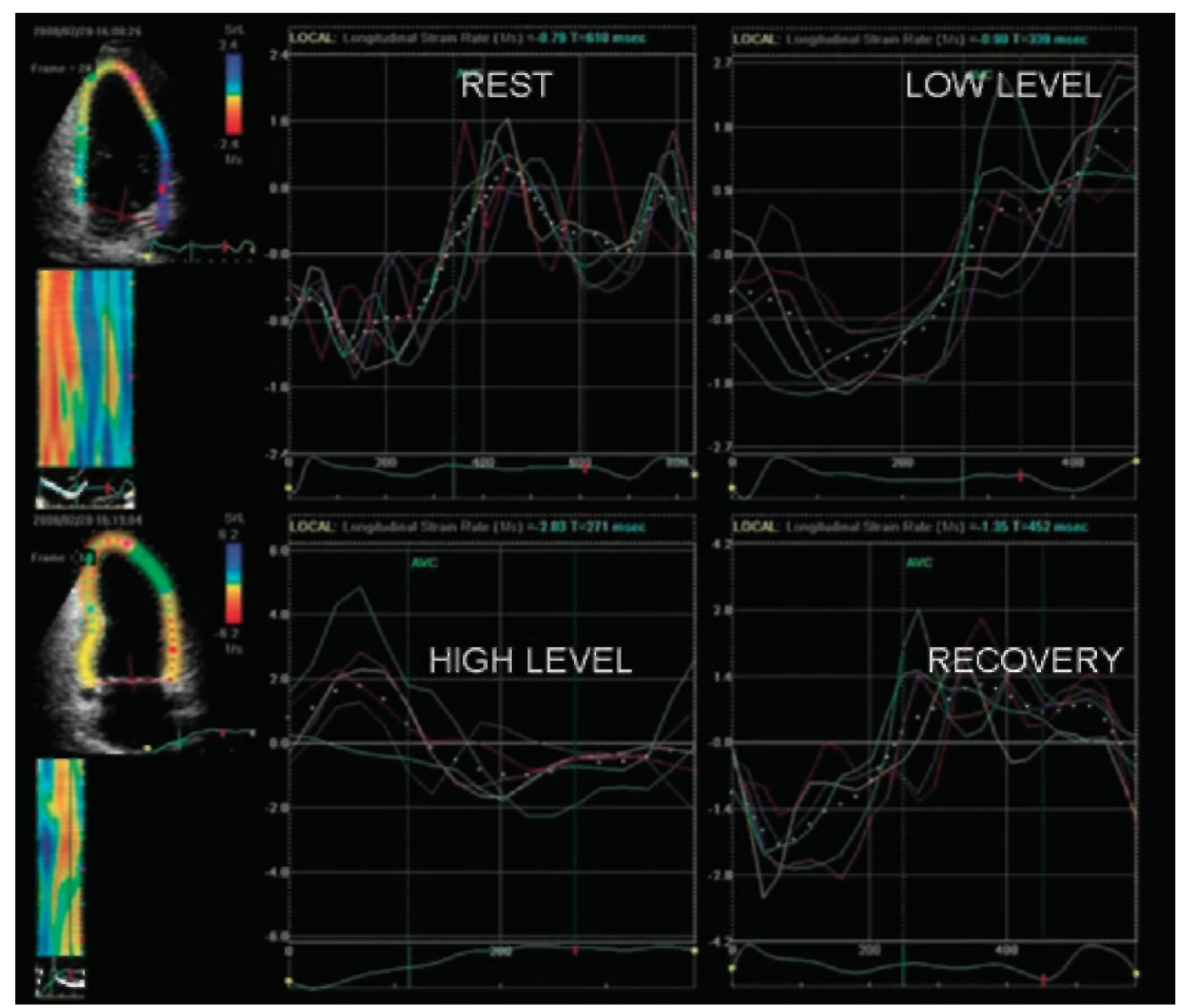

Table I- Optimal Cutoffs and Area under the Curve (AUC) of Peak Systolic Strain Rate the Diagnosis of Significant Stenosis on the Three Coronary Artery Territories

\begin{tabular}{lll}
\hline & 2D SR & TDI SR \\
\hline Cutoff LAD & $-2 / \mathrm{s}$ & $-1.7 / \mathrm{s}$ \\
AUC LAD & 0.85 & 0.87 \\
Cutoff LCx & $-1.8 / \mathrm{s}$ & $-1.6 / \mathrm{s}$ \\
AUC LCx & 0.76 & 0.78 \\
Cutoff RCA & $-1.7 / \mathrm{s}$ & $-1.3 / \mathrm{s}$ \\
AUC RCA & 0.65 & 0.83
\end{tabular}

These data are derived from only one segment SR analysis for everey coronary territory.

$\mathrm{TDI}=$ tissue Doppler imaging; $\mathrm{LAD}=$ left anterior descending artery; $\mathrm{LCx}=$ left circumflex; $\mathrm{RCA}=$ right coronary artery .

\section{STRAIN AND VIABILITY}

Myocardial viability can be determined in patients with a DSE protocol when either an improvement in resting wall motion or a bipha-sic response is observed. A biphasic response is characterized by dyssynergic myocardium at baseline that recovers contraction at low dose of dobutamine but deteriorates at higher dose because of reduced coronary flow reserve. Contractile reserve and biphasic response can also be identified during exercise if the echocardio-graphic examination is continuous. ${ }^{21,22}$ Detection of residual viable myocardial tissue within the infarct area is clinically useful and indicates a potential for functional recovery after revascularization. Evidence of functional recovery based on detection of contractile reserve with DSE is of 
comparable accuracy to alternative imaging techniques. ${ }^{23}$ Unfortunately, as discussed previously, the assessment of myocardial viability based on wall-motion scoring during DSE is difficult and subjective. Tissue Doppler, strain, and SR have been used to better quantify regional myocardial function. Hoffmann et al. ${ }^{24}$ validated the technique as a clinical tool for viability detection. Hanekom et al. ${ }^{25}$ demonstrated its interest during dobutamine stress for the prediction of functional recovery in patients undergoing revascularization after a myocardial infarction. Different parameters were defined but their sensitivity for prediction of functional recovery, was slightly better but was not significantly different from wall-motion scoring. The combination of wall motion scoring and SR parameters increased the sensitivity above wall motion scoring alone but the specificities were comparable. The obstacles in wide application of deformation imaging remain the same: complex myocardial fiber orientation, angle dependency, orientation of ultrasound beam and the need of a good acoustic window. Speckle tracking and 2D strain potentially offer a solution for these limitations. However, there are not yet clinical studies published in this setting. Figure 3 shows an example of a biphasic response during exercise in a patient with previous nontransmural infarction in the inferior and anterior walls and significant stenoses of both the right coronary artery and the LAD.

\section{CHRONIC HEART FAILURE}

New echocardiographic modalities and particularly, speckle tracking imaging seem to be a promising tool for better evaluation of the patients with heart failure. Diastolic dysfunction plays an important role in the pathophysiology of heart failure, particularly in patients presenting with symptoms of heart failure with apparently preserved LV ejection fraction (EF) and LV volumes. This condition has been classified as diastolic heart failure and, more recently, as heart failure with normal $\mathrm{EF}^{26}$ Tissue Doppler imaging has been applied in heart failure. The ratio of mitral early diastolic velocity to mitral annulus early diastolic velocity (E/Ea ratio) is currently the most practical measurement used for the estimation of LV filling pressure. Speckle-tracking echocardiography has been introduced as a method that captures the performance of all myocardial segments. Unfortunately, its use in patients suffering of systolic heart failure or diastolic heart failure has not yet been validated.

In heart failure patients, the assessment and quantification of LV dyssynchrony is clinically important to predict response to cardiac resynchronization therapy. Speckle tracking analysis has been used to measure LV dyssynchrony ${ }^{27} \mathrm{We}$ and others have previously observed that LV dyssynchrony has a dynamic component. ${ }^{28,29}$ In normal subjects, LV syn-chronicity is preserved during exercise. In contrast, in patients with systolic heart failure, LV dyssynchrony remains stable during exercise in only one-third of patients; it increases in one-third of patients and decreases in the third one-third of the population. ${ }^{29}$ These observations have been made with tissue Doppler imaging but no study has yet used the speckle tracking method to confirm and quantitate this dynamicity.

\section{EXERCISE 2D STRAIN IN VALVULAR HEART DISEASE}

The management of valvular heart disease relies upon resting evaluation only. However, most valve diseases have a dynamic component. Exercise testing can induce symptoms even in patients who deny symptoms and reveals the dynamics of the valve and the ventricle. ${ }^{30}$

\section{Mitral Regurgitation}

Asymptomatic patients with severe organic mitral regurgitation (MR) might develop latent LV contractile dysfunction despite normal LV ejection fraction. In a preliminary study we used 2D speckle tracking of longitudinal myocardial deformation at rest and during exercise. At rest, global longitudinal strain was lower in patients with MR than in normal subjects matched for age. During exercise, the increase in global strain was larger in controls than in patients. Global longitudinal strain at peak exercise was independently associated with changes in LV ejection fraction during follow-up. ${ }^{31}$

Further studies are needed to determine whether this parameter has prognostic importance.

Figure 4 shows the example of a patient with limited contractile reserve. During exercise, global longitudinal strain changed slightly; such a small increase predicts reduction in LV ejection fraction during follow-up. 
Figure 3. Strain rate $(S R)$ curves at rest and during exercise in a patient with previous anterior and inferior infarctions. A biphasic response is observed for the anterior (yellow arrows) and inferior (white arrows) segments. SR is low at rest, increases at low charge exercise and deteriorates at peak exercise.

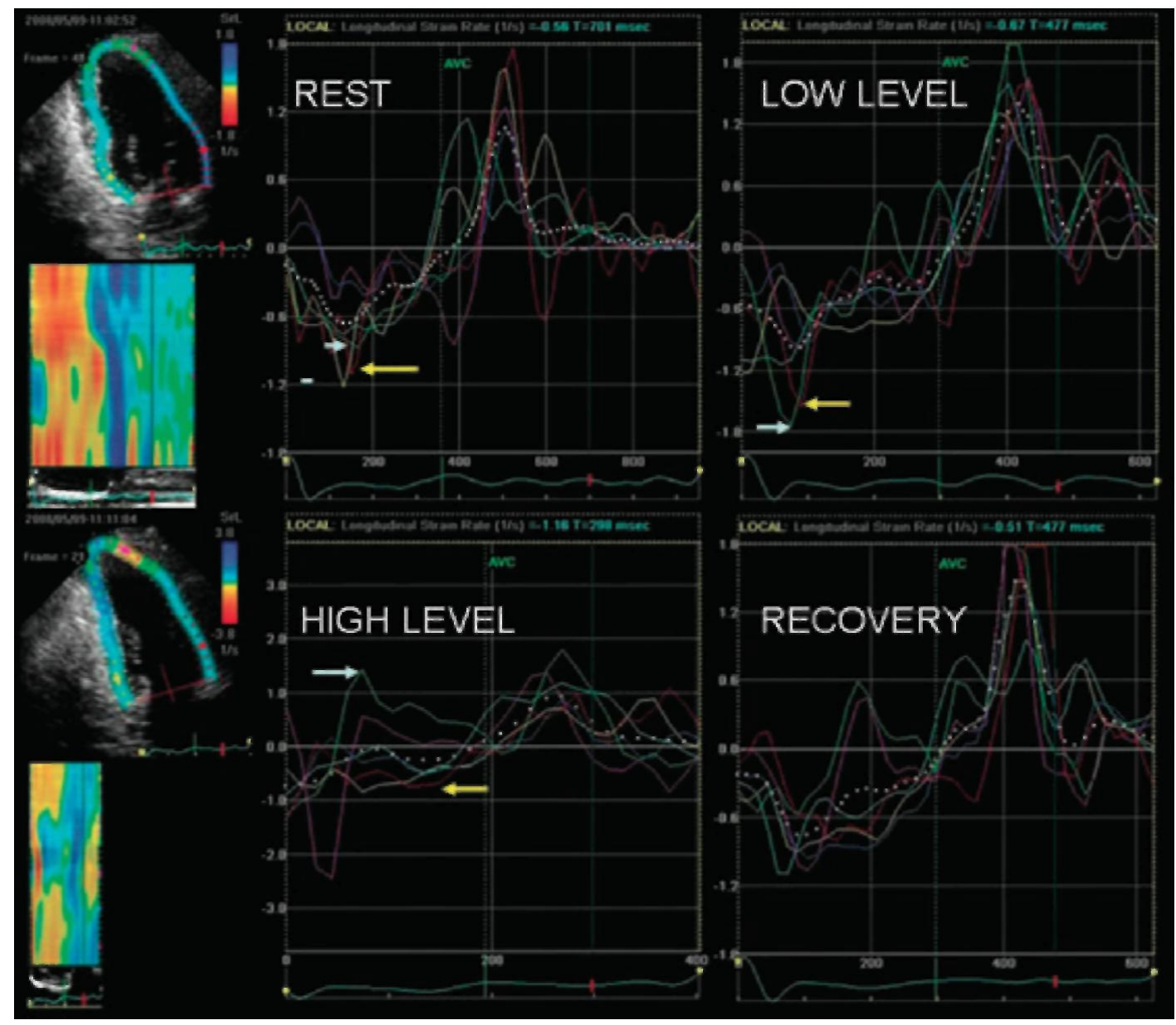

Figure 4. Images obtained at rest and during exercise in an asymptomatic patient with organic mitral regurgitation. Only small increases are observed in ejection fraction $(E F)$, peak systolic velocity (PSv) by tissue Doppler imaging and in global longitudinal 2D strain.

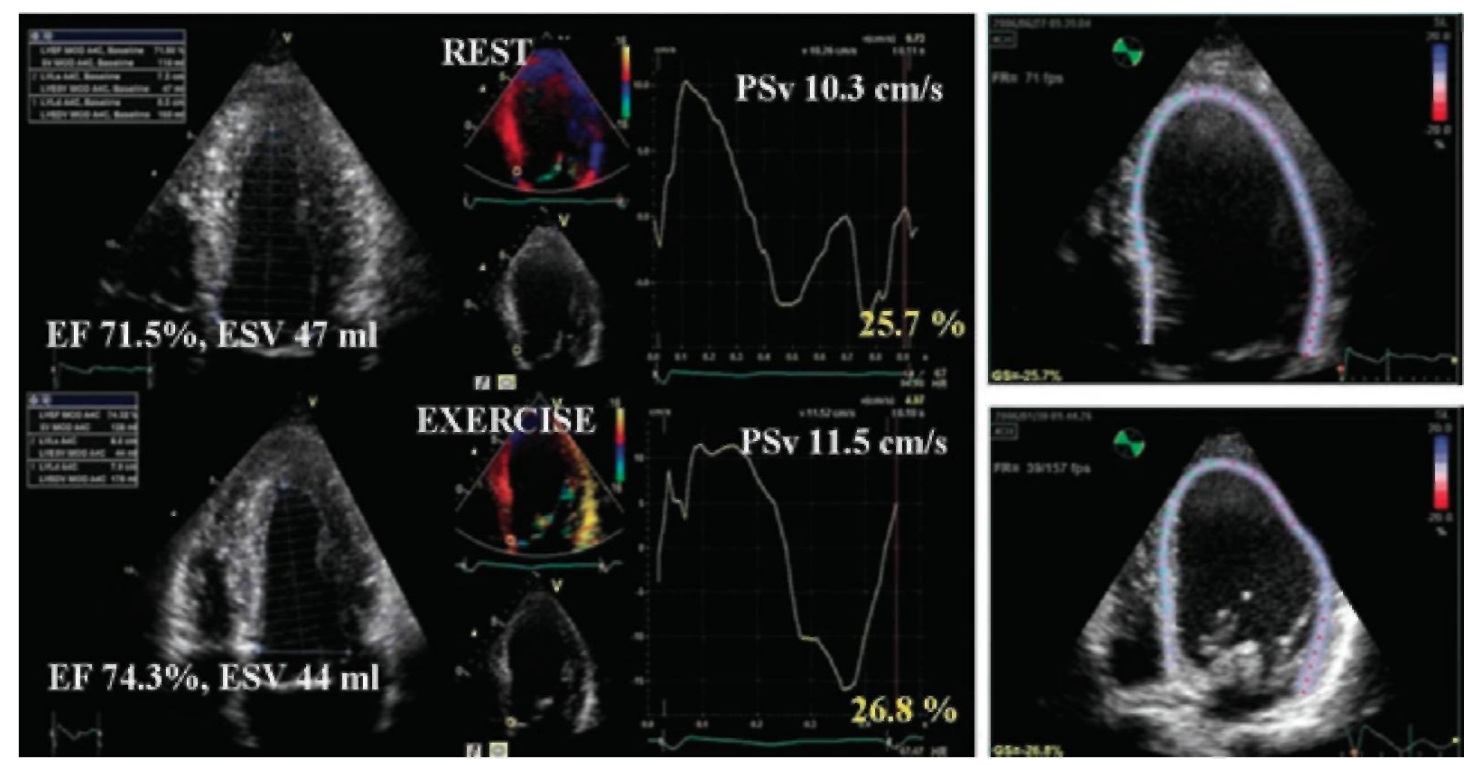


Figure 5. Absence of contractile reserve in a patient with asymptomatic severe aortic stenosis. LV ejection fraction is similar at rest and during exercise. Global longitudinal strain $(G S)$ decreases during exercise.

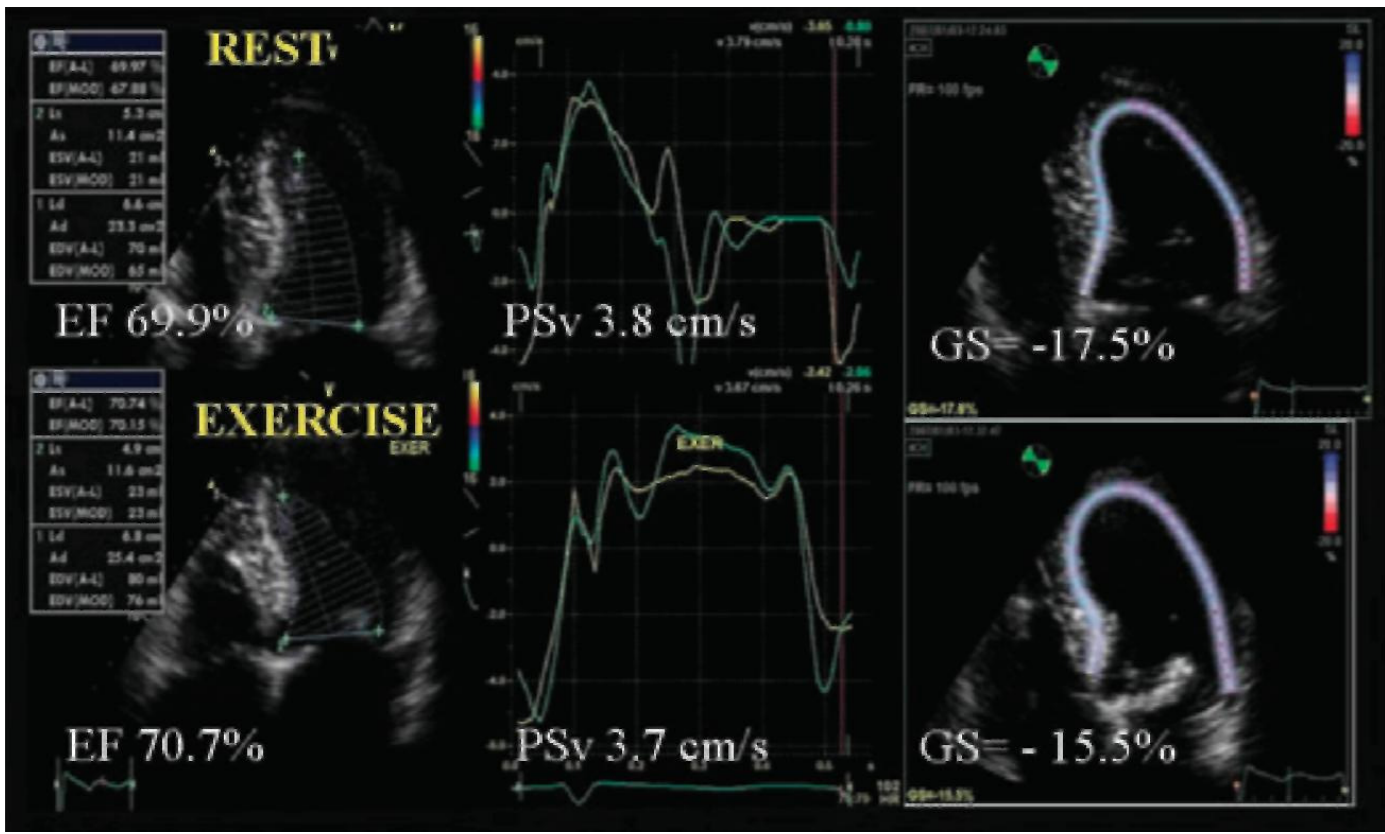

\section{Asymptomatic Aortic Stenosis}

Stress testing is recommended in asymptomatic patients with severe aortic stensosis. Currently, the management is influenced by the development of symptoms or a reduction in systolic arterial pressure during exercise. ${ }^{32}$ Global 2D longitudinal strain as determined by the speckle tracking method has the potential to provide more precise information on LV function and contractile reserve during exercise (Fig. 5). The clinical importance of these parameters remains to be evaluated.

\section{CONCLUSION}

Speckle-derived 2D strain during exercise or dobutamine stress appears to be feasible and has theoretic advantages to strain and SR obtained by tissue Doppler imaging. Preliminary studies suggest that this method coupled with stress stesting has the potential to detect inducible ischemia and contractile reserve in different clinical settings. The reliability of these easily obtainable quantitative parameters and their clinical importance will be determined by further experimental and clinical investigations.

\section{References}

1. Mason SJ, Weiss JL, Weisfeldt ML, et al: Exercise echocardiography in detection of wall motion abnormalities during ischemia. Circulation 1979;59:50-54

2. Berthe C, Pierard LA, Hiernaux M, et al: Predicting the extent and location of coronary artery disease in acute myocardial infarction by echocardiography during dobutamine infusion. Am J Cardiol 1986;58:1167-1172.

3. Picano E, Distante A, Masini M, et al: Dipyridamole-echocardiography test in effort angina pectoris. Am J Cardiol 1985;56:452-456.

4. Caidahl K, Kazzam E, Lidberg J, et al: New concept in echocardiography: Harmonic imaging of tissue without use of contrast agent. Lancet 1998;352:1264-1270.

5. Laskar R, Grayburn PA: Assessment of myocardial perfusion with contrast echocardiography at rest and with stress: An emerging technology. Prog Cardiovasc Dis 2000;43:245-258. 
Published in: Echocardiography (2009), vol. 26, iss. 3, pp. 307

Status: Postprint (Author's version)

6. Sutherland GR, Di Salvo G, Claus P, et al: Strain and strain rate imaging: A new clinical approach to quantifying regional myocardial function. J Am Soc Echocardiogr 2004;17:788-802.

7. Picano E, Lattanzi F, Orlandini A, et al: Stress echocardiography and the human factor: The importance of being expert. $J$ Am Coll Cardiol 1991;17:666-669.

8. Hoffmann R, Lethen H, Marwick T, et al: Analysis of interinstitutional observer agreement in interpretation of dobutamine stress echocardiograms. J Am Coll Cardiol 1996;27:330-336.

9. Hoffmann R, Marwick TH, Poldermans D, et al: Refinements in stress echocardiographic techniques improve inter-institutional agreement in interpretation of dobutamine stress echocardiograms. Eur Heart J 2002;23:821-829.

10. Mor-Avi V, Vignon P, Koch R, et al: Segmental analysis of color kinesis images: New method for quantification of the magnitude and timing of endocardial motion during left ventricular systole and diastole. Circulation 1997;95:2082-2097.

11. Urheim S, Edvardsen T, Torp H, et al: Myocardial strain by Doppler echocardiography. Validation of a new method to quantify regional myocardial function. Circulation 2000;102:1158-1164.

12. Voigt JU, Exner B, Schmiedehausen K, et al: Strain-rate imaging during dobutamine stress echocardiography provides objective evidence of inducible ischemia. Circulation 2003;107:2120-2126.

13. Castro PL, Greenberg NL, Drinko J, et al: Potential pitfalls of strain rate imaging: Angle dependency. Biomed Sci Instrum 2000;36:197-202

14. Perk G, Tunick PA, Kronzon I: Non-Doppler two-dimensional strain imaging by echocardiography- from technical considerations to clinical aplications. J Am Soc Echocardiog 2007;20:234-243.

15. Helle-Valle T, Crosby J, Edvardsen T, et al: New noninvasive method for assessment of left ventricular rotation: Speckle tracking echocardiography. Circulation 2005;112:3149-3156.

16. Reisner SA, Lysyansky P, Agmon Y, et al: Global longitudinal strain: A novel index of left ventricular systolic function. J Am Soc Echocardiogr 2004; 17:630-633.

17. Greenbaum RA, Ho SY, Gibson DG, et al: Left ventricular fibre architecture in man. Br Heart J 1981;45:248-263.

18. Reant P, Labrousse L, Lafitte S, et al: Experimental validation of circumferential, longitudinal, and radial 2-dimensional S during dobutamine stress echocardiography in ischemic conditions. J Am Coll Cardiol 2008;51:149-157.

19. Ingul CB, Stoylen A, Slordahl SA, et al: Automated analysis of myocardial deformation at dobutamine stress echocardiography: An angiographic validation. J Am Coll Cardiol 2007;49:1651-1659.

20. Hanekom L, Cho GY, Leano R, et al: Comparison of two-dimensional speckle and tissue Doppler S measurement during dobutamine stress echocardiography: An angiographic correlation. Eur Heart J 2007;28:1765-1772.

21. Hoffer EP, Dewe W, Celentano C, et al: Low-level exercise echocardiography detects contractile reserve and predicts reversible dysfunction after acute myocardial infarction. J Am Coll Cardiol 1999;34:989-997.

22. Lancellotti P, Hoffer EP, Piérard LA: Detection and clinical usefulness of a biphasic response during exercise echocardiography early after myocardial infarction. J Am Coll Cardiol 2003;41:1141-1147.

23. Bax JJ, Wijns W, Cornel JH, et al: Accuracy of currently available techniques for prediction of functional recovery after revascularization in patients with left ventricular dysfunction due to chronic coronary artery disease: Comparison of pooled data. $J$ Am Coll Cardiol 1997;30:1451-1460.

24. Hoffmann R, Altiok E, Nowak B, et al: Strain rate measurement by doppler echocardiography allows improved assessment of myocardial viability in patients with depressed left ventricular function. J Am Coll Cardiol 2002;39:443-449.

25. Hanekom L, Jenkins C, Jeffries L, et al: Incremental value of strain rate analysis as an adjunct to wall-motion scoring for assessment of myocardial viability by dobutamine echocardiography: A follow-up study after revascularization. Circulation 2005;112:3892-3900.

26. Sanderson JE: Heart failure with a normal ejection fraction. Heart 2007;93:155-158

27. Ypenburg C, Lancellotti P, Tops LF, et al: Acute effects of initiation and withdrawal of cardiac resyn-chronization therapy on papillary muscle dyssyn-chrony and mitral regurgitation. J Am Coll Cardiol 2007;50:2071-2077.

28. Lancellotti P, Stainier PY, Lebois F, et al: Effects of dynamic changes in left ventricular synchronic-ity on dynamic mitral regurgitation. Am J Cardiol 2005;96:1304-1307. 
Published in: Echocardiography (2009), vol. 26, iss. 3, pp. 307

Status: Postprint (Author's version)

29. Lafitte S, Bordachar P, Lafitte M, et al: Dynamic ventricular dyssynchrony: An exercise-echocardiography study. J Am Coll Cardiol 2006;47:2253-2259.

30. Piérard LA, Lancellotti P: Stress testing in valve disease. Heart 2007;93:766-772.

31. Lancellotti P, Cosyns B, Zacharakis D, et al: Importance of left ventricular function and functional reserve in patients with degenerative mitral regurgitation: assessment by two-dimensional speckle tracking. J Am Soc Echocardiog 2008;21:1331-1336.

32. Vahanian A, Baumgartner H, Bax J, et al: Task force on the management of valvular heart disease of the European Society of Cardiology: ESC Committee for Practice Guidelines. Eur Heart J 2007;28:230-268. 